\title{
A ToF-SIMS Investigation of the Thermodynamics and Bonding of Polymeric Methylene Diphenyl Diisocyanate on Oxidised Aluminium and Iron Surfaces
}

\author{
Kyoko Shimizu $^{11 *}$, Marie-Laure Abel ${ }^{1}$, Christopher Phanopoulos ${ }^{2}$, Servaas Holvoet ${ }^{2}$ and John \\ F. Watts ${ }^{1}$ \\ ${ }^{1}$ Department of Mechanical Engineering Sciences, \\ University of Surrey, Guildford, Surrey, GU2 7XH, UK \\ ${ }^{2}$ Huntsman, Everslaan 45, B-3078 Everberg, Belgium
}

\begin{abstract}
Polymeric methylene diphenyl diisocyanate (PMDI) is the major component of polyurethane formulations and as a result the adhesion, or indeed abhesion, of polyurethanes, in a variety of forms (foams, coatings and adhesives), to metal substrates will be a function of the interactions between PMDI and metal surfaces. In this paper the adsorption of PMDI on oxidised metal (aluminium and iron) substrates has been investigated. The thermodynamics of adsorption has been examined by the construction of adsorption isotherms derived from ToF-SIMS data. At low solution concentration, the adsorption isotherns of PMDI are not of the Langmuir type, but are shown to conform to Langmuir adsorption at higher solution concentrations $\left(>1 \mathrm{~g} \mathrm{~L}^{-1}\right)$. The interaction between the PMDI and iron is probably an acidbase interaction, and thus the adsorption of small PMDI molecules is displaced by larger PMDI molecules on the iron surface above a critical solution concentration. By contrast, such displacement is small on the aluminium surface as a result of dominance of covalent bond formation between PMDI and the metal substrate.
\end{abstract}

\footnotetext{
${ }^{* 1}$ Current address: Department of Chemistry, Aarhus University, DK-8000 Aarhus C, Denmark
} 


\section{Introduction}

Polyurethanes (PUs) are produced by the reaction of molecules containing an isocyanate functional group, such as polymeric methylene diphenyl diisocyanate (PMDI), with a polyol containing hydroxyl groups and commercial formulations will invariably contain a catalyst(s) and other additives. PUs exist in numerous forms ranging from flexible or rigid lightweight foams to tough, stiff, elastomers depending of their densities and precise formulation [1].

The versatile nature and varied applications of PU mean that both the ability to interact strongly (adhesion) or release readily in moulding situations (abhesion) with metals are both important in their commercial exploitation. The adhesion properties of polyurethanes to metals are critical in the production of a range of products such as car seats, furniture and laminated metal/PU panels widely used in the construction industry. The other aspect is the moulding of PU components in metal moulds where abhesion is important so that the manufacturing process can run for the required periods of time without interruption for the cleaning of the moulds and removal of debris. In previous work from this laboratory, the interfacial interactions between rigid polyurethane foams (RPUFs) and metals (aluminium and iron) have been studied by surface analysis and by employing peel testing, as well as a low-angle microtomy technique, for sample preparation prior to surface analysis [2]. In particular the nature of the chemical interaction between PMDI, the major component of a RPUF, and aluminium has been studied by XPS and ToF-SIMS. It was found that the PMDI reaction with water and/or polyol at the RPUF/metal interface was higher than that in the bulk of the foam, and that a catalyst, pentamethyldiethylenetriamine, was concentrated at the foam/metal interface. In the current paper previous work is extended to elucidate the thermodynamics of adsorption and the nature of the bonding between PMDI, used precursor for RPUF, and two widely used metal substrates.

The study of the adsorption characteristics of molecules from the liquid phase on metal surfaces is a conventional method to determine the level of polymer interaction with a solid substrate. It is straightforward to monitor the actual uptake of adsorbate on the solid surfaces by using XPS and/or ToF-SIMS. Adsorption isotherms are produced to determine the type of adsorption; whether it is chemisorption or physisorption. Physisorption forms multilayers, whereas chemisorption is limited to monolayer coverage [3]. Therefore, in the case of chemisorption, there is a plateau in the adsorption isotherm and a variety of types of chemical 
adsorption can be identified such as the Langmuir, Temkin and Freundlich types. This paper deals with the ToF-SIMS study which uses a number of fragment ions to monitor PMDI adsorption to provide a high degree of confidence in the trends established.

\section{Adsorption isotherms}

The adsorption of a solute at the solid-liquid interface can often be described by the Langmuir Equation. The Langmuir model for gas phase adsorption is based on several assumptions [4]:

- A site can be empty or occupied.

- Adsorption cannot proceed beyond monolayer coverage.

- All adsorbent sites are equivalent.

- The adsorption at particular sites is not influenced by the occupation of neighbouring sites.

- The enthalpy of adsorption is constant.

The formal description of Langmuir adsorption is treated in detail by Adamson [5]. In adsorption studies it is convenient to describe the extent of adsorption in a chemisorption process as the fractional coverage, $\theta$, which can be expressed in terms of gas pressure $(\mathrm{P})$ :

$$
\theta=\mathrm{bP} /(1+\mathrm{bP}) \quad<1>
$$

where $b$ is the ratio of the rate constants for adsorption and desorption [5], or in terms of the volume of gas adsorbed $(\Gamma)$ :

$$
\theta=\Gamma / \Gamma_{\mathrm{m}} \quad<2>
$$

where $\Gamma_{\mathrm{m}}$ is the monolayer coverage. Equation $\langle 1\rangle$ can be rearranged as:

$$
\mathrm{P} / \Gamma=\left(1 / \mathrm{b} \Gamma_{\mathrm{m}}\right)+\left(\mathrm{P} / \Gamma_{\mathrm{m}}\right) \quad<3>
$$

When adsorption occurs from the liquid phase, in which the substrate is removed from a solution of concentration $\mathrm{c}$ and the surface concentration of adsorbate $\mathrm{x}$ is measured by ToFSIMS, Equation $<3>$ can be re-written as:

$$
\mathrm{c} / \mathrm{x}=\left(1 / \mathrm{b} \Gamma_{\mathrm{m}}\right)+\left(\mathrm{c} / \Gamma_{\mathrm{m}}\right) \quad<4>
$$

Thus, Langmuir adsorption is confirmed by the linearity of the plot of $\mathrm{c} / \mathrm{x}$ versus $\mathrm{c}$. 
Not all adsorption processes follow the Langmuir model. In some cases the interaction energy becomes less negative as the extentof adsorption increases so that more energetically favourable sites are occupied first. In the Temkin isotherm, the enthalpy of adsorption decreases linearly with increasing coverage as a result of adsorbate-adsorbate interactions [7]. Whilst in the Freundlich isotherm, the enthalpy of adsorption falls logarithmically with increasing coverage because of the heterogeneity of the surface [7]. Temkin or Freundlich adsorption is confirmed by the linearity of plots of $\mathrm{x}$ versus $\ln \mathrm{c}$ or $\ln \mathrm{x}$ versus $\ln \mathrm{c}$, respectively.

The use of the surface chemical analysis methods of XPS and ToF-SIMS for the determination of adsorption isotherms from the liquid phase has been pioneered and widely used in research from this laboratory. The procedures have been fully described by Watts and Castle [4]. In the current work the diagnostic used for the assessment of adsorption type has been the linear regression factor $\left(\mathrm{R}^{2}\right)$ of the three plots indicative of Langmuir, Temkin and Freundlich adsorption. Plots of each type were constructed for each set of adsorption data and the adsorption type assigned accordingly.

\section{Role of acid-base interaction in adsorption}

The adsorption of polymers onto metal substrates from organic solvents is often dominated by acid-base interactions of the solvent, polymer and substrate surface when compounds have the ability to form acid-base interactions [8-10]. The acid-base interactions in adsorption involve the acidic or basic surface sites of the adsorbent, the acidic or basic sites of the polymer, and also often the acidic or basic sites of the solvent. The adsorption occurs only when the interaction between polymer and substrate is greater than the polymer/solvent interaction, and solvent/substrate interaction. Fowkes et al. [8] studied this triangle of acidbase interactions. For example, they studied the amount of adsorption of basic poly(methyl methacrylate) (PMMA) dissolved in basic, neutral or acidic solvents onto acidic silica [9]. They showed that the basic PMMA adsorbed onto the acidic silica most strongly from neutral solvent (following the Lewis definition) such as carbon tetrachloride (which actually is slightly acidic). As the solvent became more basic the solvents interacted with the $\mathrm{SiOH}$ sites of silica to form acid-base complexes, and hence less PMMA was adsorbed on the silica surface. Similarly it was also seen with increasing acidity of the solvent; the solvents formed 
acid-base complexes with the ester, carbonyl oxygen, groups of the PMMA. Thus, the amount of adsorption of the PMMA onto the $\mathrm{SiOH}$ sites of silica decreased.

The PMDI molecule is slightly basic as a result of the predominant basicity of the isocyanate functional groups tempered by the acidic sites of the benzene groups, although the overall the PMDI resin will exhibit acidic behaviour, as a result of the reaction of the isocyanate groups to form a polyurethane [1]. Acetone used as a solvent is also slightly basic because the oxygen atom of the carbonyl group has two lone electron pairs that can be shared with a Lewis acid [11]. Thus, the acetone solvent can interact with acidic polymers and substrates to form acid-base complexes, although in the case of PMDI solvent-solute interaction is not thought to occur to any great extent as a result of the overall basicity of the PMDI molecules, resulting from the unreacted isocynate groups. The aluminium and iron substrates used in this study were degreased with acetone to remove extraneous organic material, but an oxide/hydroxide layer will remain on all surfaces. The inevitable atmospheric exposure will also lead to the re-deposition of a very thin layer of adventitious carbon contamination. The aluminium surface is amphoteric which acts as both an electron acceptor and an electron donor $[3,12]$. Therefore, the adsorption of basic PMDI, occurs on the amphoteric aluminium surface. However, at the interface between PMDI and aluminium a covalent bond is formed, this has been established by ToF-SIMS where an ion characteristic of such a formal interaction, $\mathrm{AlCHNO}_{3}{ }^{-}$is observed in the spectra recorded from very thin films of PMDI on aluminium [13]. For this reason the interface interactions between aluminium and PMDI need to be considered in a different manner. As the iron surface is considered to be weakly acidic with an IEPS of 5.2-6.7 [14], the adsorption of the PMDI onto the iron surface is likely to occur [3].

\section{Experimental}

\subsection{Sample preparation}

Samples of $10 \times 10 \mathrm{~mm}^{2}$ were cut from aluminium (99.8\% purity) and iron (mild steel; BS 1449-Grade 4 ) sheets (Chronos Ltd Bedfordshire, UK). These coupons were then sonicated with acetone to remove organic contaminants. PMDI (Supersec® 5025, Huntsman Holland $\mathrm{BV}$, The Netherlands) solutions in the range $10^{-4}$ to $100 \mathrm{~g} \mathrm{~L}^{-1}$ were prepared. As the concentration of the solution reaches the limit at which the solution can be considered dilute, the probability of polymer-polymer interaction is increased. Therefore, the concentration of 
the solution should be below the concentration limit (i.e. dilute) to avoid the self-association of macromolecules in solution. As described above adsorption may occur as multilayers above the concentration limit as a result of the interaction of molecules with one another [15]. The concentration of the PMDI solution should be below the value of the PMDI concentration limit in order to prevent PMDI self-association. The value was calculated using the approach of Kurata et al [16] which yield a value of $600-800 \mathrm{~g} \mathrm{~L}^{-1}$ depending on the value of the radius of gyration of PMDI $(0.49-0.53 \mathrm{~nm})$ employed in the calculation [17]. The upper concentration of the solutions used in this study is $100 \mathrm{~g} \mathrm{~L}^{-1}$, well below the PMDI concentration limit determined above. A $100 \mathrm{~g} \mathrm{~L}^{-1}$ stock solution of the PMDI was prepared by dissolving $10 \mathrm{~g}$ of the PMDI in acetone in a $100 \mathrm{ml}$ volumetric flask. Subsequently, the solution was diluted to provide a set of solutions, decreasing in PMDI concentration in a stepwise manner by an order of magnitude from $10^{2}$ down to $10^{-4} \mathrm{gL}^{-1}$. The structure of the PMDI molecule is presented in Figure 1. Aluminium and iron samples were immersed in 40 $\mathrm{cm}^{3}$ of the chosen solution for 20 minutes to establish kinetic equilibrium [4]. The samples were then immediately rinsed with fresh acetone three times for 2 minutes and left to drain in a vertical orientation onto aluminium foil. The samples were left on aluminium foil to dry in the laboratory atmosphere for at least 1 hour.

\subsection{ToF-SIMS analysis}

ToF-SIMS analysis was achieved using a TOF.SIMS 5 (ION-TOF GmbH, Münster, Germany). Static SIMS conditions with a total ion dose less than $1 \times 10^{13}{\text { ions } \mathrm{cm}^{-2} \text { analysis }}^{-1}$ were employed using a $9.5 \mathrm{keV} \mathrm{Bi}_{3}{ }^{+}$primary ion beam operating in the high current bunched mode for high spectral resolution (which is greater than $10^{4}$ at mass $m / z=29 u^{\text {If }}$ ) [18]. An analysis area of $100 \times 100 \mu \mathrm{m}^{2}$ at a resolution of $64 \times 64$ pixels was used. ToF-SIMS spectra were acquired over a mass range of 1-850 $\mathrm{u}$ in both positive and negative ion modes. Charge compensation was achieved using a pulsed electron flood source. Fragments of known composition, at low and high masses were used for mass calibration, including those characteristic of aluminium, iron and the PMDI, to ensure mass scale linearity across the mass range of interest.

\footnotetext{
${ }^{\pi} \mathrm{u}$ is the unified atomic mass unit defined as one twelfth of the mass of one atom of ${ }^{12} \mathrm{C}$.
} 
The ToF-SIMS intensities for particular fragment ions under consideration are evaluated using the concept of relative peak intensity (RPI), which is the ratio of the intensity of the ion of interest relative to the total ion intensity from $m / z=1$ to $850 \mathrm{u}$ [19]

$$
\mathrm{RPI}_{\mathrm{x}}=\mathrm{I}_{\mathrm{x}} / \mathrm{I}_{\text {total }} \quad\langle 13>
$$

where $\mathrm{x}$ is the ion of interest and $\mathrm{I}_{\text {total }}$ is the total ion intensity between $\mathrm{m} / \mathrm{z}=1$ and $850 \mathrm{u}$, and $I_{x}$ is the measured intensity of the ion under consideration. The data presented is the mean of four measurements on the same sample. The error bars included on all data represent plus/minus one standard deviation, although in some cases the error bars are so tight they are obscured by the graphical symbols.

\section{Results}

ToF-SIMS spectra were acquired for all metal samples treated with PMDI. Several characteristic fragments of the PMDI molecule in the positive ToF-SIMS spectrum $(\mathrm{at} \mathrm{m} / \mathrm{z}=$ 106, 132, 197, 206, 223, and 328 u) were selected to study the adsorption of the PMDI. These fragments were shown in previous work to be diagnostic of PMDI, the complete fragmentation scheme being shown in [13]. The high resolution regions of the mass spectrum, over a region of $0.3-0.5 \mathrm{u}$ around the peak of interest, are shown in Figure 2. Here the benefits of operating in the high spectral resolution ToF-SIMS mode are clearly seen with peaks separated by less than $0.1 \mathrm{u}$ being readily resolvable. The assignment of the peaks is based on comparison of the experimentally recorded mass (following mass calibration), $\mathbf{M}_{\text {meas, }}$, with that of the theoretical (exact) mass of the candidate fragment ion, $\mathbf{M}_{\text {ext }}$. The accuracy of this assignment is expressed as a parameter, $\Delta$, in parts per million where:

$$
\Delta=\left(\left[\mathrm{M}_{\text {meas }}-\mathrm{M}_{\text {ext }}\right] / \mathrm{M}_{\mathrm{ext}}\right) \times 10^{6}
$$

An assignment was assumed to be correct when $\Delta \leq 50 \mathrm{ppm}$. As a check on the fidelity of ion assignment and the reliability of each ion assigned to PMDI to predict adsorption, the behaviour of each ion, on either the iron or aluminium substrate, was followed as a function of solution concentration. Figure 3 shows this behaviour as the ToF-SIMS uptake of characteristic PMDI ions by plotting the RPI of each fragment as a function of the solution concentration. The background levels at which these fragments are detected on the reference samples (degreased metal substrates, without any adsorption treatment) are indicated by horizontal dashed lines. The adsorption isotherms of all fragments exhibit the same trends for each substrate, not only reinforcing the origin of all as being from the PMDI molecule, 
but also indicating that the behaviour of the iron samples is very different to that of the aluminium samples. The uptake of all PMDI fragments on aluminium increase very quickly at highly dilute solution concentration and decreases slightly at $10 \mathrm{~g} \mathrm{~L}^{-1}$ concentration, and then reaches a plateau. For the iron samples, the uptake of all PMDI fragments also rises rapidly and reaches a plateau at $1 \mathrm{~g} \mathrm{~L}^{-1}$ concentration, followed by an increase ahead of a second plateau.

\section{Discussion}

\subsection{General Adsorption Characteristics}

There are a number of characteristics that influence the adsorption of PMDI on metal surfaces and in this section the overall behaviour is discussed and comparison drawn with work that describes similar adsorption isotherm shapes. The manner in which the homologue type influences adsorption is discussed and the behaviour at very low concentrations, which may influence a degree of site selectivity in these solutions, considered in terms of a change from one adsorption type to another at a critical solution concentration. Finally constants associated with Langmuir adsorption are deduced from the experimental data presented in $\S 5$.

The PMDI adsorption on the aluminium and iron at very low concentration exhibit similar trends, the uptakes of the PMDI rise rapidly up to $1 \mathrm{~g} \mathrm{~L}^{-1}$ concentration and reach a plateau for iron and a maximum for aluminium. This adsorption behaviour shows typical chemisorption indicating that all the potential adsorption sites on these substrates are occupied by adsorbate molecules [4, 20-25]. The adsorption of PMDI on aluminium then slightly drops at $10 \mathrm{~g} \mathrm{~L}^{-1}$ concentration and then reaches a plateau, whilst the PMDI adsorption on iron increases again above $10 \mathrm{~g} \mathrm{~L}^{-1}$ concentration. The PMDI solution is well below the concentration limit and can be considered dilute and thus no self-association of the PMDI molecules will occur and adsorption appears to be limited to one monolayer. This ensures that the changes in conformation of the adsorbed PMDI molecules result from the interaction between the PMDI and the metal substrate rather than the PMDI molecules themselves [12]. There are several important factors in studying adsorption of polymers: the molecular weight and molecular weight distribution, orientation of the polymer on the substrate, the rate of adsorption, the nature of the interaction, porosity and heterogeneity of the substrate surface, and environmental factors such as temperature, solvent composition and 
$\mathrm{pH}[12-15,26]$. The most significant property affecting this phenomenon is molecular weight together with molecular weight distribution.

\subsection{The Phenomenon of Oscillatory Adsorption}

The adsorption behaviour on the aluminium sample, that is the increase, decrease and a very gradual increase in adsorption, as a function of solution concentration, is reminiscent of the kinetic oscillatory adsorption behaviour that has been described by Quinton et al. [27, 28], who studied the kinetic behaviour of organosilanes on iron and aluminium oxide surfaces [28-31]. An example from the work of these authors is reproduced in Figure 4, and the similarity in shape between these kinetic data and the thermodynamic data of Figure 2 is readily seen. This oscillatory behaviour was explained by the presence of several different species, monomer, dimer and larger molecules, in solution, and was described in the following manner: Rapid adsorption of monomer units, then desorption of these adsorbed monomer units via displacement by water molecules and irreversible slow adsorption of dimers or larger species via a condensation reaction with hydroxyl groups on the metal surface. This phenomenon is also seen in studies of the thermodynamics (concentration) of polymer adsorption, and explains why the polydispersity of the polymer adsorbate can have a marked effect on adsorption behaviour. Thomsen et al also studied the kinetics of adsorption and the orientation behaviour of both short- and long-chain alkoxy-based organosilanes on aluminium oxide surfaces [27]. The oscillatory adsorption system was independent of alkyl chain length of the molecules. They showed that the degree of molecular alignment correlated with the molecular packing density on the aluminium oxide surface. At low adsorption times, the molecular density was maximised and the molecules exhibited the greatest degree of alignment. Subsequently, the surface density was minimised as a result of increase of the intermolecular separations of the alkyl chains, and the adsorbed film becomes disordered. As adsorption time increases, the molecular density increases again with the increasing degree of molecular alignment.

A major difference between the work of Quinton et al. and this work is that they studied the kinetic behaviour of adsorption while in this work the thermodynamics of adsorption has been studied, kinetic equilibrium having been attained by the choice of an extended adsorption time. In previous work using this approach it has been shown that kinetic equilibrium is reached in approximately five minutes, in the current studies an adsorption time of twenty minutes has been employed. However, their interpretation of the result is 
useful in developing a hypothesis to explain the phenomenon later. Grilli et al. [32] studied the adsorption of an epoxy acrylate resin on aluminium alloy with two different commercial conversion coatings: one based on chromium(VI) and a chromium-free alternative commercially available as Nabutan ${ }^{\circledR}$ [32]. They found that the adsorption of the epoxy acrylate resin on non-chromium (VI) pre-treatment exhibited a concentration oscillatory behaviour. The resin used in their work is based on diglycidyl ether of bisphenol A (DGEBA) and iso-bornyl acrylate (IBA), and competitive adsorption between these two molecules in Nabutan ${ }^{\circledR}$ leads to an oscillatory adsorption phenomenon. Both molecules are adsorbed up to a critical concentration with the IBA molecules adsorbed at the expense of the DGEBA molecules. As the concentration increases the DGEBA molecules are adsorbed, displacing IBA. Below a critical concentration, the small and rigid IBA molecules (compared with DGEBA) have a better chance to adsorb onto the substrate via the acrylate functionality. As the solution concentration increases, the relatively flexible DGEBA molecules have more affinity for the substrate with a plethora of bonding sites.

\subsection{The Effect of PMDI Homologue Type}

The PMDI used in this work contains a mixture of di-iso $(n=2)$, tri-iso $(n=3)$ and small amounts of higher homologues of MDI [1,33]. The smaller molecules, which have lower molecular weight, are preferentially adsorbed initially on the substrate, as they arrive at the solid surface sooner than larger ones as a result of their more ready diffusion in the liquid solution. The reduction in the surface concentration of carbon and increase of nitrogen after immersion in the $0.0001 \mathrm{~g} \mathrm{~L}^{-1}$ solution of PMDI from XPS data (not shown here [17]), indicates that the physisorbed adventitious carbon was displaced by the PMDI molecules. The newly arrived larger molecules are then adsorbed on the substrate if there are still available adsorption sites on the substrate $[15,32]$. All adsorption sites are occupied by the small molecules initially, subsequently the small molecules can be displaced by the larger molecules, which would form a more stable and durable bond with the metal surface as a consequence of the greater number of bonding groups per molecule [15, 32]. The fragment at mass 328 u contains one isocyanate group and two amine groups as shown in Figure 1, which are formed when isocyanate groups react with hydroxyl groups of the polyol. Referring to the schematic of PMDI presented in Figure 1 this fragment originates from tri-iso or higher homologue species (i.e. $n \geq 3$ ). The increase of the intensity of fragment at mass $328 \mathrm{u}$ after the knee of the isotherm for iron samples indicates that there may be displacement of the remaining smaller molecules by the larger molecules. For aluminium samples, however, the 
decrease of the intensity of fragment $(\mathrm{m} / \mathrm{z}=328 \mathrm{u})$ at $10 \mathrm{~g} \mathrm{~L}^{-1}$ concentration implies that no such displacement occurs after the knee of the uptake curve, and apparent changes in uptake may be a result of changes in molecular conformation [12]. In general terms, polymer adsorption on the solid surfaces of the substrate at low concentrations, is mainly dominated by small molecules, but at higher concentrations these are displaced by larger molecules. This pre-supposes that adsorption takes place in equilibrium with desorption in the usual manner. When the surfaces are covered by a monolayer of PMDI molecules, small and large molecules are adsorbed on the substrate surfaces and fill all available adsorption sites.

After monolayer coverage, aluminium and iron surfaces exhibit a different PMDI adsorption behaviour. In the case of aluminium samples, only small molecules are adsorbed initially on the substrate surface. Because the smaller molecules have higher kinetic energies and diffusion coefficients than the larger molecules, they will arrive sooner at the surface than the large ones [34]. However, the PMDI molecules can form a covalent bond with the aluminium surface [13]. Therefore these small molecules cannot be displaced by larger molecules because of the existence of a strong interfacial interaction. As only small molecules of PMDI are adsorbed on the surface, a decrease of intensity of PMDI molecules after the point of monolayer coverage is observed. However, the intensity of the PMDI rises slightly and reaches a second plateau at higher concentration, and thus there is a small amount of displacement of small molecules by larger molecules. This indicates that the interfacial interaction is not only through covalent bonding but also that a small amount of other interactions such as Lewis acid-base interactions are present (in which case, some of the small molecules can be displaced by larger molecules).

For the PMDI adsorption on iron surface, the intensities of the PMDI molecules exhibit a significant increase after reaching monolayer coverage, followed by a second plateau. The interaction between the PMDI and the iron is relatively weak so that the small molecules that are adsorbed can be readily displaced by larger molecules. The larger molecules tend to adsorb more strongly than the small molecules, as they have more functional groups for anchoring the molecule, thus when they arrive on the substrate surface they displace the small molecules $[5,31]$.

Another possible reason for the second plateau at high solution concentration for both the aluminium and the iron samples is an increase in the packing density of the PMDI molecules 
$[12,27,35]$. The density of the PMDI molecules is low in the highly diluted solution, and thus the molecules are more likely adsorbed in random or parallel to the surface to interact with one or both sides of isocyanate group. In the more concentrated solutions, the molecular conformation can become more compact allowing an increase in the density of adsorbed molecules. Therefore, the molecular alignment is more likely to be perpendicular, and this change in conformation of the adsorbed molecules may lead to a second plateau for iron samples, as observed for PMMA molecules adsorbed on oxidised iron and silicon substrates [12]. On the contrary, such change in molecular conformation cannot happen at the interface as a result of the covalent bond formation between aluminium and PMDI.

\subsection{Behaviour at Low PMDI Concentrations}

The extent to which the adsorption data presented in Figure 3 conform to the simplest adsorption models can be established using the methods described in $\$ 2$ to produce Langmuir, Temkin and Freundlich plots, although as the analysis presented above implies, this is a relatively simplistic approach searching for a common feature in the adsorption characteristics. Data that conforms to a particular isotherm type will yield a linear relationship and consequently a least squares regression value of unity $\left(R^{2}=1\right)$. This approach provides a straightforward manner in which to assess which adsorption model best fits the experimental data set, by choosing the data set with a least squares regression value closest to unity. However the response to the Langmuir test is heavily biased by the linear abscissa which effectively biases the test towards the higher concentrations and gives little weight to the cluster of low concentration data points. The plots for PMDI fragment ions 106 $\mathrm{u}$ and $132 \mathrm{u}$ are presented in Figure 5. The linear regression values deduced from these plots are presented in Table 1.

Over the entire concentration range the PMDI adsorption isotherms may conform to Langmuir, Temkin or Freundlich type. Table 1 shows the comparison of the $\mathrm{R}^{2}$ values obtained from these plots. Most data show tolerable agreement with all adsorption types but the Langmuir plot fits well for all PMDI fragments; thus it is confirmed that the uptake of PMDI on substrates is best described by Langmuir adsorption across the entire concentration range. However at the lower solution concentrations the fit to the Langmuir model is not as good and below PMDI concentrations of $1 \mathrm{gL}^{-1}$ the data follows a logarithmic relationship for both substrates. This is very clearly seen in Figure 5 (c) and (d) where the data of Figure 5 (a) and (b), which show Langmuir adsorption well, is re-plotted on logarithmic axes. The 
solid lines on Figure $5(\mathrm{a}-\mathrm{d})$ represent Langmuir behaviour. The regression values for double logarithm plots, Figure $5(\mathrm{~g})$ and $(\mathrm{h})$, at concentrations below $1 \mathrm{gL}^{-1}$ are in the range of $0.997-1.000$ for ions characteristic of PMDI on iron surfaces and $0.960-1.000$ for aluminium. This is indicative of non-Langmuir behaviour such as Temkin or Freundlich adsorption, and the situation for iron is particularly clear, on the basis of these $\mathrm{R}^{2}$ values. At higher concentrations the fit to the Langmuir Equation is good and thus the regression value for the linear plot is close to unity. The explanation for this apparent contradiction may be found by consideration of the conformation of the molecules in solution. As discussed above, the PMDI used in this work contains a mixture of homologues of MDI, and initially the smaller molecules seek out the more favourable sites first (which, of course, is contrary to the Langmuir model which assumes all sites are equivalent and explains the non-Langmuir behaviour at low concentrations), thus data at the lowest concentrations conform to a model in which, unlike the Langmuir model, more favourable sites exist for adsorption and the exothermic enthalpy of adsorption reduces as such sites become occupied. As more concentrated solutions are used to probe the surface, such small molecules may become displaced, as described above, and the overall behaviour at concentrations greater than $1 \mathrm{gL}^{-1}$ takes on a Langmuir form.

\subsection{Langmuir Constants for the Adsorption of PMDI on Aluminium and Iron}

The Langmuir plots are made by plotting the value of c/RPI versus c. The graph is linear, which gives the slope, $1 / \Gamma_{\mathrm{m}}$, and intercept, $1 / \Gamma_{\mathrm{m}} b$, and thus, the calculated equilibrium coverage values of $\Gamma_{\mathrm{m}}$ and $b$, which is a constant related to the enthalpy of adsorption on aluminium and iron, are shown in Table 2. Iron substrate exhibits higher $\Gamma_{\mathrm{m}}$ values than aluminium substrate and this indicates that the iron surface has a larger concentration of adsorption sites for PMDI than aluminium surfaces. Aluminium samples, however, exhibit a greater $b$ value than the iron samples. The constant $b$ may be defined, taking $a$ thermodynamic approach, by [12]:

$$
b=\frac{[\bar{v} \sigma n(\theta-1) M !]}{\theta Z m v M \Gamma \exp (-Q / R T)} \quad<14>
$$

where $\bar{v}$ is the velocity component of a molecule perpendicular to the substrate surface, $\sigma$ is the sticking coefficient per bond of macromolecule on substrate, $\mathrm{n}$ is the degree of polymerization, $(\theta-1)$ is the proportion of available substrate adsorption sites, $Z m$ is the number of substrate per unit area, $v$ is the vibration frequency of adsorbed atoms within 
macromolecules, $\Gamma$ is the surface concentration of the macromolecule, $\mathrm{M}$ is the maximum number of bonds formed between adsorbed molecules and substrate, and Q is the interaction energy per mole of bonds of adsorbed molecule with the substrate [12]. The constant $b$ is conveniently described by:

$$
\mathrm{b}=\mathrm{b}_{\Phi} \exp (\mathrm{Q} / \mathrm{RT}) \quad<15>
$$

The constant $b_{\Phi}$ contains a stereochemistry factor as well as a frequency factor, and thus the value of $b$ can be affected by the differences in stereochemistry and in the interaction energy.

The molecular conformation of PMDI on the aluminium surface is random and will tend to be parallel to the surface, while it is more likely to be perpendicular on the iron surface and this leads to a higher $\Gamma_{\mathrm{m}}$ value than for the aluminium sample. The high $\mathrm{b}$ value of aluminium samples is a result of the interaction energy of the covalent bond that is formed between the PMDI adsorbate and the aluminium substrate, which is higher than that of the acid base interaction of the PMDI and iron surface.

\section{Conclusions}

The adsorption of the PMDI has been studied in order to understand the interaction between PMDI with aluminium and iron substrates. The uptakes of PMDI components on both aluminium and iron substrates form monolayer coverage at low concentration, and in spite of the overall agreement with the Langmuir model adsorption, at concentrations below $1 \mathrm{~g} \mathrm{~L}^{-1}$ chemisorption is of a non-Langmuir type indicating small molecules adsorbing to the more advantageous sites at these lower solution concentrations. Unfortunately it is not possible to unambiguously assign adsorption behaviour to a specific model in this range of concentrations, but the deviation from the Langmuir model is striking and leads to the important conclusion above. The PMDI contains a mixture of di-iso, tri-iso and higher homologues and initially all potential adsorption sites on these substrates are occupied by small molecules, subsequently being displaced by larger molecules at higher concentrations. The interaction between the PMDI and iron is probably an acid-base interaction, and thus the small molecules can be displaced by the larger molecules on the iron substrate at higher concentrations. For aluminium samples, however, such a displacement is small after the monolayer coverage as a result of covalent bond formation between the PMDI and aluminium. A small increase of the PMDI components indicates that the interface interaction 
is not just covalent bond formation; there might be other interactions such as acid-base interactions.

\section{Acknowledgements}

The authors thank Huntsman PU Everberg Belgium for financial support by way of a studentship to KS, and Professor Jim Castle for invaluable discussion on surface adsorption.

\section{$\underline{\text { References }}$}

1. D. Randall and S. Lee, The Polyurethanes Book, John Wiley \& Sons Ltd, Chichester (2002).

2. K. Shimizu, M.-L. Abel, C. Phanopoulos, S. Holvoet and J. F. Watts, J. Mater. Sci., 47, 902-918 (2012).

3. A. J. Kinloch, Adhesion and Adhesives: Science and Technology, (Chapman and Hall, Cambridge UK, 1995) Chapters 3 and 6.

4. J. F. Watts and J. E. Castle, Int. J. Adhes. Adhes., 19, 435-443 (1999).

5. A. W. Adamson, Physical Chemistry of Surfaces $5^{\text {th }}$ ed., (John Wiley \& Sons Ltd, New York 1990).

6. A. Aeimbhu, J. E. Castle and P. Singjai, Surf. Interf. Anal., 37, 1127-2236 (2005).

7. I. D. Mall, V. C. Srivastava and N. K. Agarwal, J. Hazard. Mater., 143, 386-395 (2007).

8. F. M. Fowkes, J. Adhes. Sci. Tech., 1, 7-27 (1987).

9. F. M. Fowkes and M. A. Mostafa, Ind. Eng. Chem. Prod. Res. Dev., 17, 3-7 (1978).

10. M. Marmo, H. Jinnai, M. A. Mostafa. F. M. Fowkes and J. A. Manson, Ind. Eng. Chem. Prod. Res. Dev., 15, 206-211 (1976).

11. D. E. Packham, Handbook of Adhesion $2^{\text {nd }}$ Edition., D. E. Packham (Ed.) (John Wiley \& Sons Ltd, Chichester, West Sussex 2005) pp.10-12.

12. J. F. Watts, S. R. Leadley, J. E. Castle and C. J. Blomfield, Langmuir, 16, 2292-2300 (2000).

13. K. Shimizu, C. Phanopoulos, R. Loenders, M-L. Abel and J. F. Watts, Surf. Interf. Anal., 42, 1432-1444, (2010).

14. G A Parks, Chem Rev, 65, 177-198 (1965). 
15. J. J. Kipling, Adsorption from Solutions of Non-electrolytes, (Academic Press, London 1965) Chapters 3, 7-8 and 10.

16. K. Kurata and Y. Tsunashima, Polymer Handbook $3^{\text {rd }}$ ed., J. Brandup and E. H. Immergut (Eds.) (Wiley, New York 1989), pp.VII/1-5.

17. K Shimizu, PhD Thesis, University of Surrey, 2011

18. R. A. De Souza, J. Zehnpfenning, M. Martin and J. Maier, Solid State Ionics, 176, 1465-1471 (2005).

19. J. F. Watts, A. Rattana and M-L. Abel, Surf. Interf. Anal., 36, 1449-1468 (2004).

20. J. F. Watts, Adhesion - Current Research and Application, W. Possart (Ed.) (WileyVCH Verlag GmbH \& Co, KGaA, Weinheim, Germany 2005), pp.1-16.

21. M-L. Abel, M. M. Chehimi, A. M. Brown, S. R. Leadley and J. F. Watts, J. Mater. Chem., 5(6), 845-848 (1995).

22. P. E. Vickers, M. E. Turner, M-L. Abel and J. F. Watts, Composites A, 29A, 1291-1304 (1998).

23. P. E. Vickers, J. E. Castle and J. F. Watts, Appl. Surf. Sci., 150, 244-254 (1999)

24. A. Rattana, J. D. Hermes, M-L. Abel and J. F. Watts, Int. J. Adhes. Adhes., 22, 205-218 (2002).

25. A. Rattana, M.-L. Abel and J. F. Watts, Int. J. Adhes. Adhes., 26, 28-39 (2006)

26. D. Myers, Surfaces, Interfaces and Colloids: Principles and Applications, (VCH Publishers, New York 1991) Chapters 9 and 14.

27. L. Thomsen, B. Watts, D. V. Cotton, J. S. Quinton and P. C. Dastoor, Surf. Interf. Anal., 37, 472-477 (2005).

28. J. S. Quinton, L. Thomsen and P. C. Dastoor, Surf. Interf. Anal., 25, 931-936 (1997).

29. J. S. Quinton, P. C. Dastoor and W. Allison, Surf. Sci., 402-404, 66-70 (2000).

30. J. S. Quinton and P. C. Dastoor, Surf. Interf. Anal., 30, 25-28 (2000).

31. J. S. Quinton and P. C. Dastoor, Surf. Interf. Anal., 32, 57-61 (2001).

32. R. Grilli, M.- L. Abel, M. A. Baker, B. Dunn and J. F. Watts, Int. J. Adhes. Adhes., 31, 687-694 (2011).

33. T. Gruke, "New Advances in Polymeric MDI Variants", EUROCOAT 2002, Barcelona, Spain (June 2002) p.3.

34. G. P. van der Beek, M. A. Cohen Stuart and G. J. Fleer, Macromolecules, 24, 35533561 (1991).

35. J. F. Watts, A. Rattana and M-L. Abel, Surf. Interf. Anal., 36, 1449-1468 (2004). 
Table 1: Tests for adsorption type: Comparison of the least square values $\left(\mathrm{R}^{2}\right)$ for characteristic fragments of PMDI treated substrates for Langmuir, Temkin and Freundlich adsorption.

\begin{tabular}{rccccc}
\hline & 106 & 132 & 197 & 206 & 223 \\
\hline \hline Aluminium & & & & & \\
Langmuir & 0.988 & 0.976 & 0.992 & 0.994 & 0.990 \\
Temkin & 0.711 & 0.590 & 0.566 & 0.621 & 0.548 \\
Freundlich & 0.725 & 0.716 & 0.662 & 0.707 & 0.658 \\
Iron & & & & & \\
Langmuir & 0.976 & 0.980 & 0.986 & 0.972 & 0.987 \\
Temkin & 0.706 & 0.739 & 0.719 & 0.669 & 0.748 \\
Freundlich & 0.920 & 0.939 & 0.861 & 0.878 & 0.899 \\
\hline
\end{tabular}

Table 2: $\Gamma_{\mathrm{m}}$ and $\mathrm{b}$ values obtained from the Langmuir plots

\begin{tabular}{rccccc}
\hline & 106 & 132 & 197 & 206 & 223 \\
\hline \hline Aluminium & & & & & \\
$\Gamma_{\mathrm{m}}\left(\mathrm{x} \mathrm{10}^{-4}\right)$ & 116.2 & 26.9 & 4.47 & 4.0 & 2.4 \\
$\mathrm{~b}$ & 0.63 & 0.58 & 1.79 & 1.03 & 2.51 \\
Iron & & & & & \\
$\Gamma_{\mathrm{m}}\left(\mathrm{x} \mathrm{10}^{-4}\right)$ & 312.3 & 70.3 & 13.6 & 11.3 & 8.8 \\
$\mathrm{~b}$ & 0.26 & 0.29 & 0.39 & 0.27 & 0.39 \\
\hline
\end{tabular}




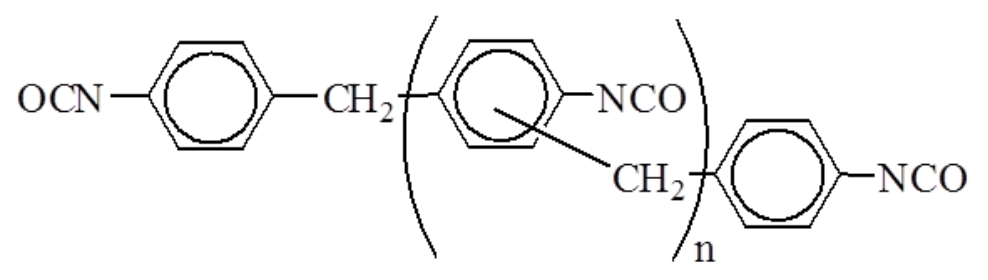

Figure 1: Structure of the PMDI 
(a)

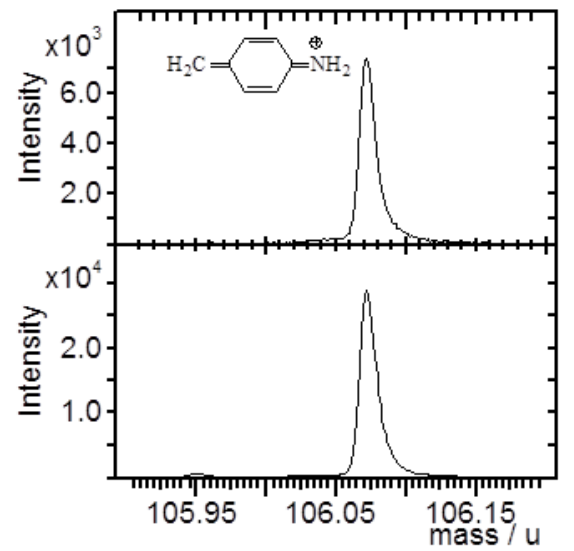

(c)

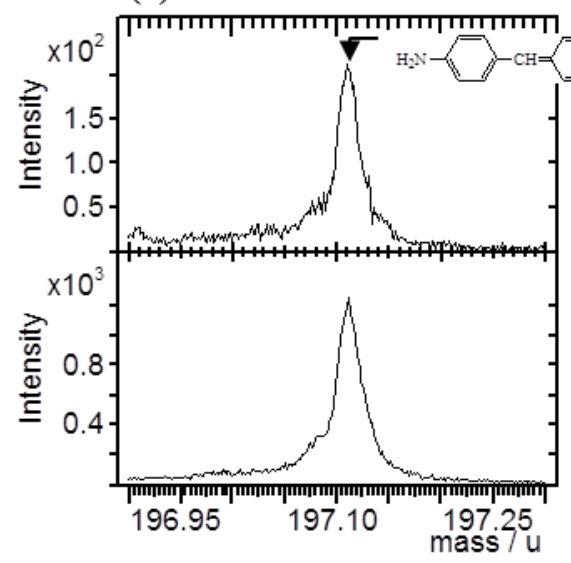

(e)

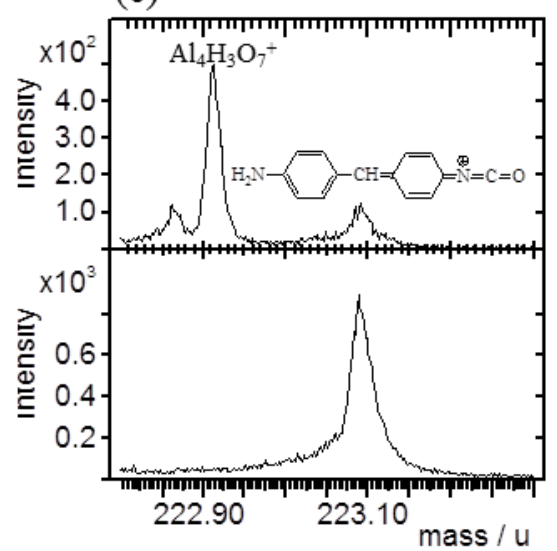

(b)

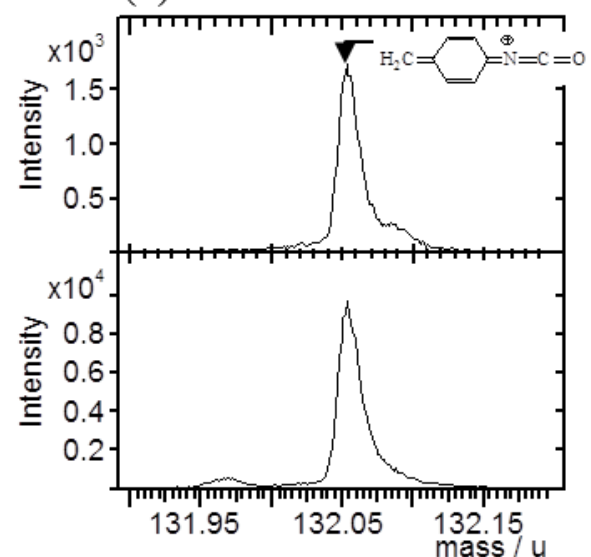

(d)

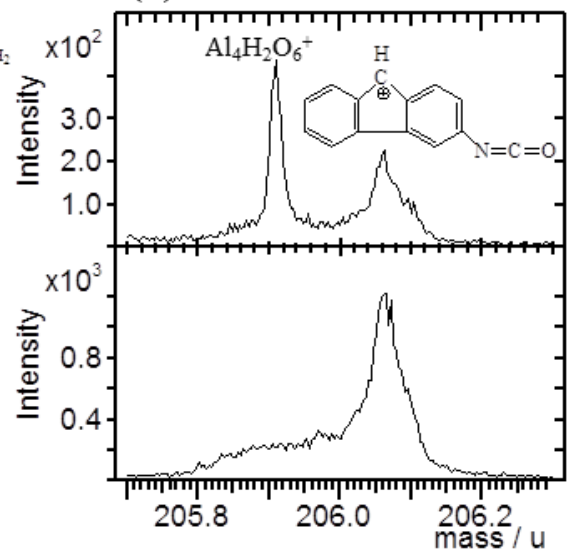

(f)

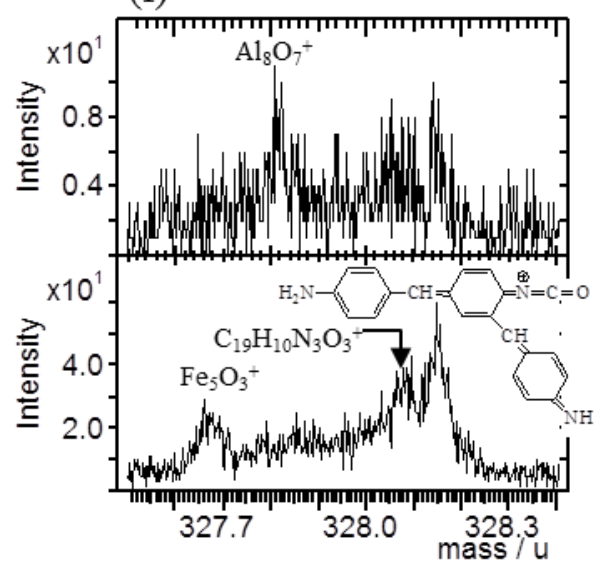

Figure 2: Positive ToF-SIMS spectra at high resolution of aluminium (top) and iron (bottom) treated with $100 \mathrm{~g} \mathrm{~L}^{-1}$ PMDI solutions for nominal masses (a) $106 \mathrm{u}$, (b) $132 \mathrm{u}$, (c) $197 \mathrm{u}$, (d) $206 \mathrm{u}$, (e) $223 \mathrm{u}$ and (f) $328 \mathrm{u}$. The structures of the ions diagnostic of PMDI are shown for each pair of spectra. 
(a) Mass $106 \mathrm{u} \quad \stackrel{\oplus}{\stackrel{\oplus}{\mathrm{N}}=}=\mathrm{CH}_{2}$

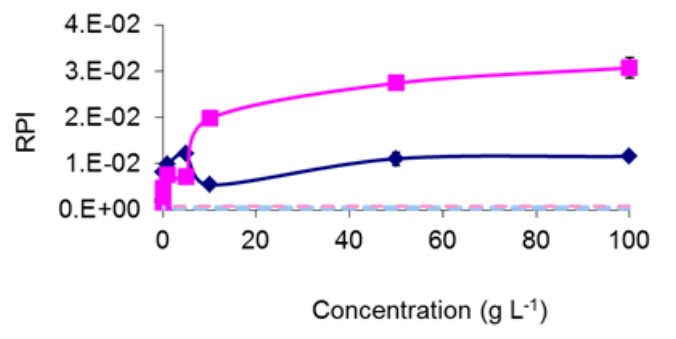

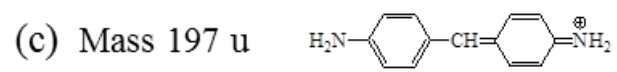

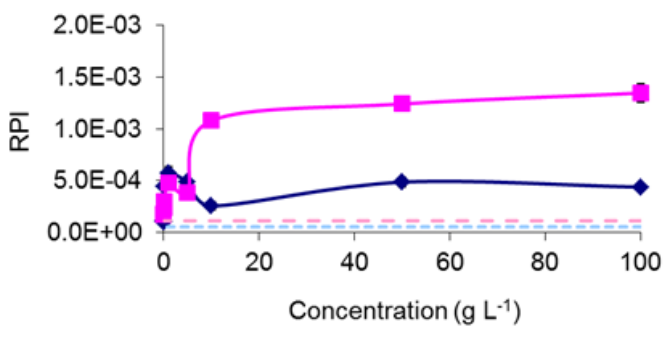

(e) Mass $223 \mathrm{u} \quad \mathrm{H}_{2} \mathrm{~N}-\longrightarrow-\mathrm{CH}=\longrightarrow=\mathrm{N}=\mathrm{C}=\mathrm{O}$

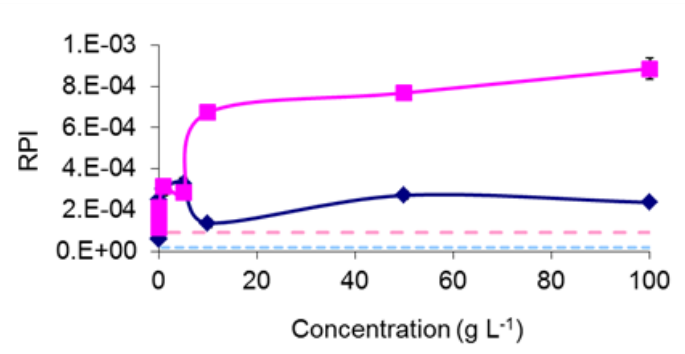

(b) Mass $132 \mathrm{u} \quad \mathrm{O}=\mathrm{C}=\stackrel{\oplus}{=}=\mathrm{CH}_{2}$

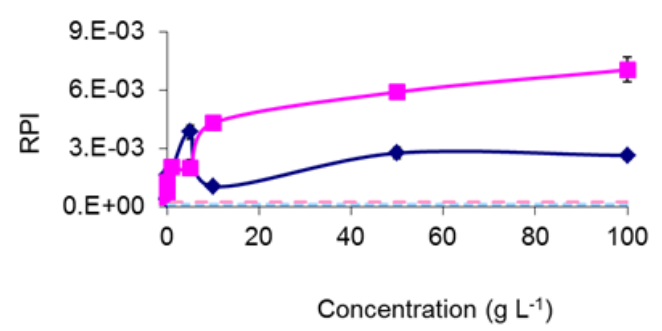

(d) Mass $206 \mathrm{u}$
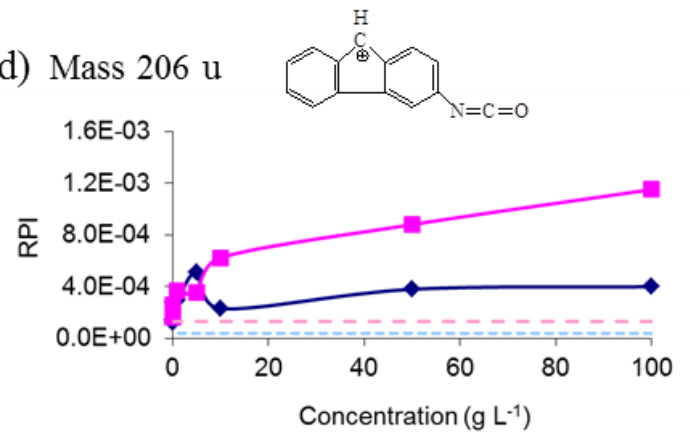

(f) Mass $328 \mathrm{u}$

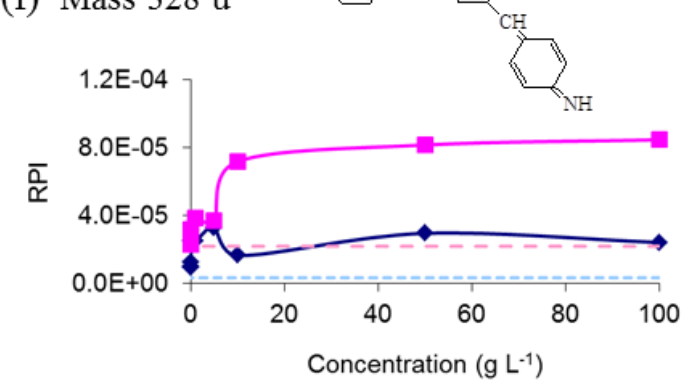

Figure 3: Uptake of PMDI on aluminium ( $\bullet$ and iron ( $\bullet$ ) surfaces, using the fragments (a) $106 \mathrm{u}$, (b) $132 \mathrm{u}$, (c) $197 \mathrm{u}$ (d) $206 \mathrm{u}$ (e) $223 \mathrm{u}$ and (f) $328 \mathrm{u}$ from ToF-SIMS positive spectra. Aluminium reference (--) and iron reference (--), intensities (RPIs) are shown with dotted lines. 


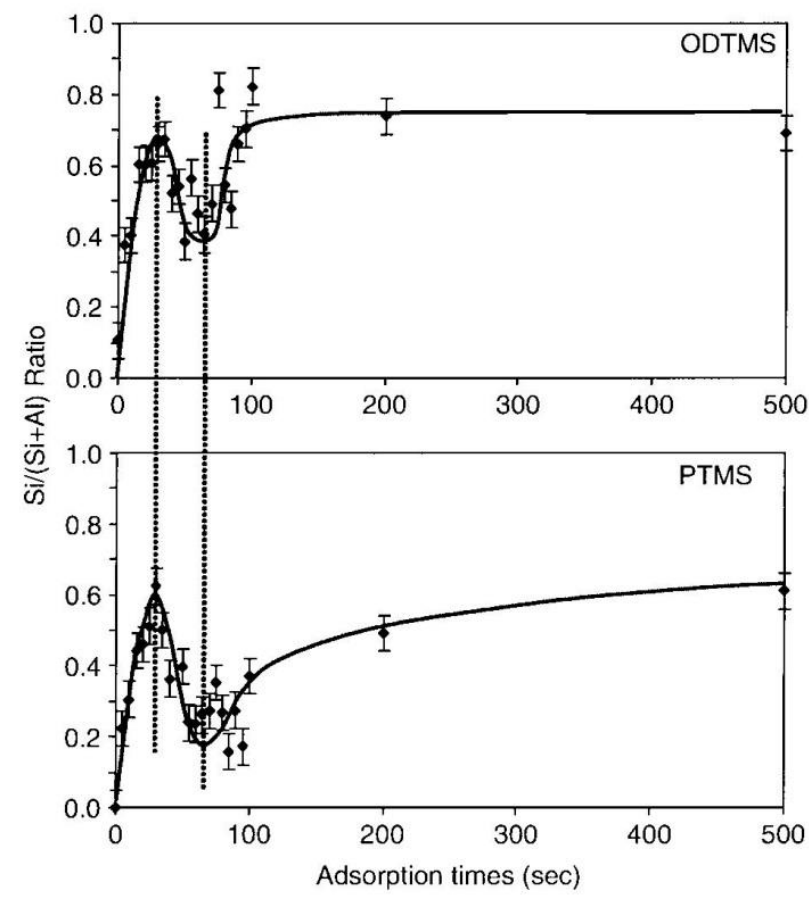

Figure 4. An example of kinetic oscillatory adsorption from the work of Thomsen et al [27]. The two adsorption isotherms represent the adsorption of octadecyltrimethoxysilane (ODTMS) and the shorter chain propyltrimethoxysilane (PTMS) from $0.75 \%$ solution (acidified ethanolic solution $+10 \%$ water for ODTMS and an acidified aqueous solution in the case of PTMS) on aluminium. The vertical dashed lines serve to highlight the oscillatory adsorption phenomenon. Reproduced from [27] by permission of John Wiley and Sons Ltd. 

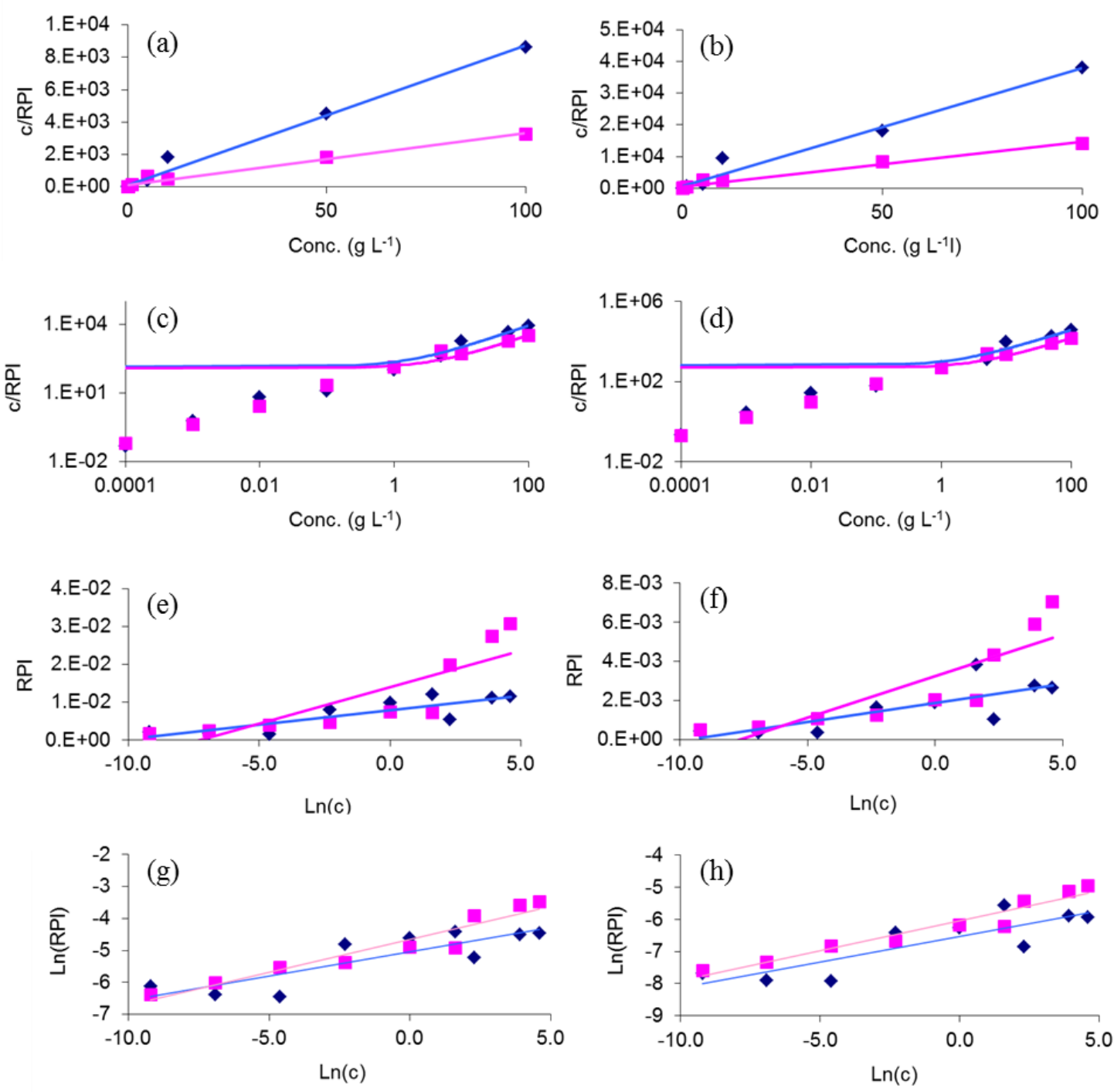

Figure 5. Langmuir plots of the PMDI adsorbed on to aluminium and iron substrates, from the from the fragments (a) $106 \mathrm{u}$ and (b) $132 \mathrm{u}$, Langmuir plots of (a) and (b) transposed to double logarithmic axes (c) and (d), Temkin plots from the fragments (e) $106 \mathrm{u}$ and (f) $132 \mathrm{u}$ and Freundlich plots from fragments (g) $106 \mathrm{u}$ and (h) $132 \mathrm{u}$. The solid lines represent Langmuir, Temkin or Freundlich behaviour (as appropriate) for aluminium (blue) and iron (pink) surfaces, data points are for PDMI adsorption on aluminium ( $\bullet$ ) and iron ( $\square$ ) surfaces. 